\title{
GPU accelerated spectral finite elements on all-hex meshes
}

\author{
J.-F. Remacle ${ }^{\mathrm{a}, \mathrm{b}}$, R. Gandham ${ }^{\mathrm{a}}$, T. Warburton ${ }^{\mathrm{a}}$ \\ ${ }^{a}$ Department of Computational and Applied Mathematics, Rice University \\ ${ }^{b}$ Université catholique de Louvain, Institute of Mechanics, Materials and Civil \\ Engineering (iMMC), Bâtiment Euler, Avenue Georges Lemaître 4, 1348 \\ Louvain-la-Neuve, Belgium
}

\begin{abstract}
This paper presents a spectral element finite element scheme that efficiently solves elliptic problems on unstructured hexahedral meshes. The discrete equations are solved using a matrix-free preconditioned conjugate gradient algorithm. An additive Schwartz two-scale preconditioner is employed that allows h-independence convergence. An extensible multi-threading programming API is used as a common kernel language that allows runtime selecti on of different computing devices (GPU and CPU) and different threading interfaces (CUDA, OpenCL and OpenMP). Performance tests demonstrate that problems with over 50 million degrees of freedom can be solved in a few seconds on an off-the-shelf GPU.

Keywords: Spectral Finite Elements, GPU computing, Hexahedral Meshes
\end{abstract}

\section{Introduction}

Recent research efforts [1, 23] have led to the development of 3D hexdominant mesh generation systems that are fast and reliable. It is now possible (e.g. with Gmsh [10]) to create meshes of general 3D domains that contain over $80 \%$ of hexahedra in volume in a fully automatic manner.

We foresee that fully automatic hex-meshing procedures will be available in the next decade. This perspective allows finite element researchers to reconsider some commonly held beliefs, namely that tet-meshing may not remain the only solution for automatic mesh generation.

Email addresses: jean-francois.remacle@uclouvain.be (J.-F. Remacle), rajeshgandham@gmail.com (R. Gandham), tim.warburton@gmail.com (T. Warburton) 
Quadrilateral meshes in 2D and hexahedral meshes in 3D are usually considered to be superior to triangular/tetrahedral meshes. There are numerous modeling reasons to prefer hexes: boundary layers in CFD [22], inaccuracy or locking problems in solid mechanics [2].

From a high order spectral finite element perspective, hex meshes provide considerable advantages. First, although this is not specific to spectral finite elements, a hex mesh contains about seven times fewer elements than a tet mesh with the same number of vertices. Fewer elements mean less data storage and a faster assembly procedure. Taking advantage of the inherent tensor-product structure of hexahedral basis functions one can dramatically reduce the number of floating point operations for computing finite element operators. The local cartesian structure of the mesh provides natural overlapping patches of elements that enables the construction of efficient local preconditioners. Finally, spectral hex-meshes can achieve relatively high throughput on GPUs following the approaches described below.

The use of GPUs for accelerating finite element solvers for elliptic problems is of course not new. In early work Göddeke et al [13] investigated scalability of finite element solvers on GPU clusters. Later Göduke described multigrid methods for finite element methods on GPU clusters [12. Cecka et al [3] and Markall et al [16] discussed algorithms for efficient stiffness matrix assembly on GPUs. Knepley et al [15] described algorithms for efficient evaluation of finite element integrals on GPUs. Gaveled et al [11] introduced a finite element toolkit that integrates geometric multigrid techniques with sparse approximate inverse algorithms on GPUs. Furthermore, pushing the envelope of GPU based finite element software design $\mathrm{Fu}$ et al [7] describe a systematic approach to pipelining finite element methods. Largely these prior approaches have focused on optimizing the process of stiffness matrix assembly. The current work differs by first using a high-order finite element approach and secondly adopting a matrix-free approach that in its leanest form only requires storage for mesh vertex coordinates, residual vector, solution vector, load vector, and indexing arrays.

In this paper, we propose a numerical scheme that allows us to solve Poisson-like problems on unstructured all-hex meshes using the massive multi-threading capacities of modern computer hardware. An extensible multi-threading programming API is used as a common kernel language [17] to try our numerical scheme on different devices (GPU and CPU) and using different thread programming interfaces (CUDA, OpenCL, and OpenMP).

This paper is structured as follows. In \$2, standard properties of spectral finite elements are presented in brief. The numerical method is presented in $\$ 3$ and $\S 4$ : preconditioned conjugate gradients are used for solving linear 
systems. A two-scale additive Schwartz preconditioner is used for accelerating the convergence. Details of implementation are presented in $\$ 5$ and results are presented in $\$ 6$.

\section{Spectral Finite Elements on Hexahedral Meshes}

Consider a domain $\Omega \in R^{3}$ with boundary $\Gamma=\Gamma_{D} \cup \Gamma_{N}$ and the following model problem: find $u(x, y, z)$ that satisfies

$$
\begin{array}{rll}
c u-\nabla \cdot(\kappa \nabla u)=s & \text { on } & \Omega, \\
u=u_{0} & \text { on } & \Gamma_{D} \\
\frac{\partial u}{\partial n}=g & \text { on } & \Gamma_{N}
\end{array}
$$

where $c(x, y, z)>0, \kappa(x, y, z)>0$ and $s(x, y, z)$ is a given source term. We further suppose that $s, u_{0}$ and $g$ satisfy the standard regularity assumptions and, without loss of generality, that $u_{0}=0$. A weak formulation of (3) is: find $u \in H_{0}^{1}(\Omega)$ that satisfies

$$
\int_{\Omega}[\kappa \nabla u \cdot \nabla w+c u w] d x d y d z=\int_{\Omega} r w d x d y d z \quad \forall w \in H_{0}^{1}(\Omega)
$$

where $H_{0}^{1}(\Omega)=\left\{u \in H^{1}(\Omega),\left.u\right|_{\Gamma}=0\right\}$.

\subsection{Interpolation}

Consider now a mesh constructed of unstructured hexahedra. On each hexahedron $e$, the finite element interpolation basis is a tensor products of one dimensional basis of $P_{n}$ that are the set of Lagrangian interpolants $\phi_{j}(t), j=0, \ldots, n$ on the Gauss-Lobatto Legendre (GLL) quadrature points in the reference domain: $t_{i} \in[-1,+1], i=0, \ldots, n, \phi_{j}\left(t_{i}\right)=\delta_{i j}$ [4].

In the reference hexahedron $\xi, \eta, \zeta \in[-1,+1]$ of element $e$, fields are interpolated as

$$
u^{e}(\xi, \eta, \zeta)=\sum_{i=0}^{n} \sum_{j=0}^{n} \sum_{k=0}^{n} u_{i j k ; e} \phi_{i}(\xi) \phi_{j}(\eta) \phi_{k}(\zeta)
$$

where $u_{i j k ; e}$ are the values of $u$ at the $(n+1)^{3}$ nodes of element $e$. We define the derivation matrix $D$ following [4] as

$$
D_{i j}=\left.\frac{d \phi_{i}}{d t}\right|_{t=t_{j}} .
$$




\subsection{Local and Global vectors}

Consider a mesh made of $N_{E}$ unstructured hexahedra with a total of $N$ GLL nodes and a scalar field $u$ interpolated on the mesh. In the following, two representations of $u$ will be used, one that is defined locally to one element and a second that is defined globally on the mesh. The local version of $u$ is denoted by

$$
u_{i j k ; e}, \quad 0 \leq i, j, k \leq n, \quad 1 \leq e \leq N_{E}
$$

A global indexing of the GLL nodes is defined that associates a unique number to every GLL node $\mathcal{N}$ of the mesh. The global version of $u$ is noted

$$
u_{\mathcal{N}}, \quad 1 \leq \mathcal{N} \leq N
$$

A local-to-global indexing table $\mathcal{N}=I_{l \rightarrow g}(i j k ; e)$ provides a global index $\mathcal{N}$ for local node $(i j k ; e)$. A scatter operation [6] consists of building the local representation of a vector from its global representation:

$$
u_{i j k ; e}=u_{I_{l \rightarrow g}(i j k ; e)} .
$$

The gather operator allows to compute global vectors from local vectors. It requires the definition of a global-to-local indexing table $I_{g \rightarrow l}(\mathcal{N})$ that returns the $N_{\mathcal{N}}$ local nodes $\left(i_{\mathcal{N}_{l}} j_{\mathcal{N}_{l}} k_{\mathcal{N}_{l}} ; e_{\mathcal{N}_{l}}\right)$ that are associated to a given global node $\mathcal{N}$ :

$$
I_{l \rightarrow g}\left(I_{g \rightarrow l}(\mathcal{N})\right)=I_{l \rightarrow g}\left(i_{\mathcal{N}_{l}} j_{\mathcal{N}_{l}} k_{\mathcal{N}_{l}} ; e_{\mathcal{N}_{l}}\right)=\mathcal{N}, \quad l=1, \ldots, N_{\mathcal{N}}
$$

\subsection{Geometry}

The unstructured nature of the hexahedral meshes that are considered here force us to consider the geometry of each individual element $e$. The geometry of element $e$ is defined through its mapping

$$
x^{e}(\xi, \eta, \zeta), \quad y^{e}(\xi, \eta, \zeta), \quad z^{e}(\xi, \eta, \zeta)
$$

between the reference cube $\xi, \eta, \zeta \in[-1,+1]$ and the element $e$. We define the Jacobian

$$
J^{e}(\xi, \eta, \zeta)=\left[\begin{array}{lll}
\frac{\partial x^{e}}{\partial \xi} & \frac{\partial x^{e}}{\partial \eta} & \frac{\partial x^{e}}{\partial \zeta} \\
\frac{\partial y^{e}}{\partial \xi} & \frac{\partial y^{e}}{\partial \eta} & \frac{\partial y^{e}}{\partial \zeta} \\
\frac{\partial z^{e}}{\partial \xi} & \frac{\partial z^{e}}{\partial \eta} & \frac{\partial z^{e}}{\partial \zeta}
\end{array}\right],
$$

its determinant $|J|^{e}=\operatorname{det} J^{e}$ and the symmetric metric tensor $G^{e}=\left(J^{e}\right)^{T} J^{e}$. 


\subsection{Integration}

In the spectral element method formulation GLL points are both used for interpolation and integration purposes:

$$
\int_{e} f(x, y, z) d x d y d z \simeq \sum_{i=0}^{n} \sum_{j=0}^{n} \sum_{k=0}^{n} f_{i j k ; e} \underbrace{\rho_{i} \rho_{j} \rho_{k}|J|_{i j k ; e}}_{m_{i j k ; e}}
$$

where the $\rho_{j}$ 's are $1 D$ integration weights. GLL points are sub-optimal integration points: they only allow to exactly integrate a polynomial of order $2 n-1$

The computation of (4) is performed in two steps. Local values of the residuals are computed at every GLL point of every element:

$$
r_{i j k ; e}=m_{i j k ; e}\left[\left.\left.\kappa_{i j k ; e} \nabla u\right|_{i j k ; e} \cdot \nabla\left(\phi_{i} \phi_{j} \phi_{k}\right)\right|_{i j k ; e}+c_{i j k ; e} u_{i j k ; e}\right] .
$$

Then local residuals $r_{i j k ; e}$ are gathered to global GLL nodes $\mathcal{N}$ using (8) as

$$
r_{\mathcal{N}}=\sum_{l=1}^{N_{\mathcal{N}}} r_{i_{\mathcal{N}} j_{\mathcal{N}} k_{\mathcal{N}} ; e}
$$

Equations (5) and (6) allow to compute

$$
\left.\frac{\partial u}{\partial \xi}\right|_{i j k ; e}=\sum_{m=0}^{n} D_{i m} u_{m j k ; e},\left.\quad \frac{\partial u}{\partial \eta}\right|_{i j k ; e}=\sum_{m=0}^{n} D_{j m} u_{i m k ; e},\left.\quad \frac{\partial u}{\partial \zeta}\right|_{i j k ; e}=\sum_{m=0}^{n} D_{k m} u_{i j m ; e} .
$$

Thus, $r_{i j k ; e}$ is computed as

$$
\begin{array}{ll}
\sum_{m=0}^{r_{i j k ; e}}= & m_{i j k ; e}[ \\
\sum_{m=0}^{n} & \kappa_{m j k ; e} D_{m i}\left[\left.G_{m j k ; e}^{1} \frac{\partial u}{\partial \xi}\right|_{m j k ; e}+\left.G_{m j k ; e}^{2} \frac{\partial u}{\partial \eta}\right|_{m j k ; e}+\left.G_{m j k ; e}^{3} \frac{\partial u}{\partial \zeta}\right|_{m j k ; e}\right]+ \\
\sum_{m=0}^{n} & \kappa_{i m k ; e} D_{m j}\left[\left.G_{i m k ; e}^{2} \frac{\partial u}{\partial \xi}\right|_{i m k ; e}+\left.G_{i m k ; e}^{4} \frac{\partial u}{\partial \eta}\right|_{i m k ; e}+\left.G_{i m k ; e}^{5} \frac{\partial u}{\partial \zeta}\right|_{i m k ; e}\right]+ \\
& \kappa_{i j m ; e} D_{m k}\left[\left.G_{i j m ; e}^{3} \frac{\partial u}{\partial \xi}\right|_{i j m ; e}+\left.G_{i j m ; e}^{5} \frac{\partial u}{\partial \eta}\right|_{i j m ; e}+\left.G_{i j m ; e}^{6} \frac{\partial u}{\partial \zeta}\right|_{i j m ; e}\right]+
\end{array}
$$




\section{Preconditioned Conjugate Gradients}

The aim now is to solve our problem, i.e. find $u$ solution of

$$
r_{\mathcal{N}}(u)=0, \quad 1 \leq \mathcal{N} \leq N
$$

For that, we use preconditioned conjugate gradients (PCG) because of the symmetric positive definite nature of our problem. Algorithm 1 describes the $\mathrm{PCG}$ procedure. In this description, $\mathcal{P}$ is a preconditioner. The two

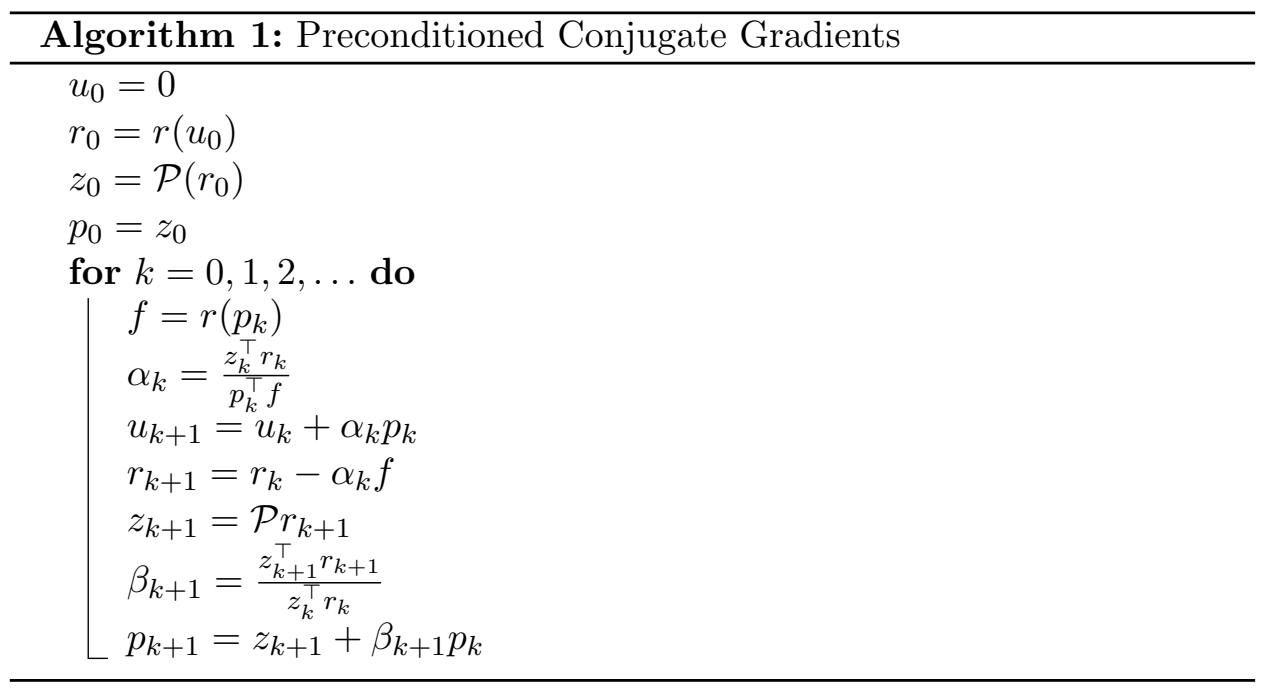

expensive steps that are computed at each iteration are actually computing $r\left(p_{k}\right)$ and computing $\mathcal{P} r_{k+1}$.

\section{A two scale preconditioner}

We use here an additive Schwarz preconditioner $P$ using overlapping subdomains [5] plus a coarse grid projection operator. Preconditioner $\mathcal{P}$ is based on solving (i) a coarse problem with low order $(n=1)$ elements on the mesh and (ii) local problems on overlapping subdomains. The contributions of the coarse and fine preconditioners are subsequently added:

$$
\mathcal{P}=\mathcal{P}^{c}+\mathcal{P}^{f}
$$




\subsection{Coarse grid preconditioner}

In short, the coarse grid preconditioner works as follows

$$
\mathcal{P}^{c} r=V^{T} \mathcal{P}_{0} V r .
$$

$V$ is a restriction operator that projects the high order residual onto the coarse space. In our implementation, $\mathcal{P}_{0}$ consists of algebraic multigrid cycles on the finite element matrix computed at polynomial order $n=1$. The prolongation operator $V^{T}$ is chosen as the transpose of $V$ in order to preserve the symmetry of the problem.

The essential ingredient of this multilevel is the restriction operator. In [14] authors show that $L^{2}$ projection is a good choice for $V$. In that specific case:

$$
V=C m^{-1}
$$

where $m$ is the fine scale mass matrix and where $C$ the "mixed" mass or correlation matrix. Note that $m$ is the lumped diagonal mass matrix when using spectral finite elements: the $m_{\mathcal{N}}$ 's are the global components of the local weights $m_{i j k ; e}$ :

$$
m_{\mathcal{N}}=\sum_{l=1}^{N_{\mathcal{N}}} m_{i_{\mathcal{N}} j_{\mathcal{N}} k_{\mathcal{N}} ; e} .
$$

The application of $\mathcal{P}^{c}$ to the residual $r$ proceeds as:

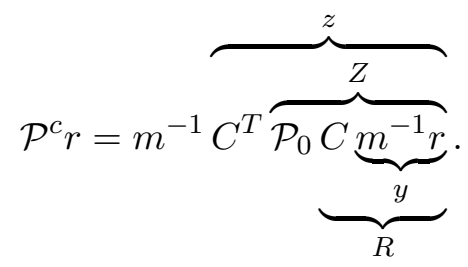

Assume that capital letters indices $I, J$ and $K$ are coarse indices: $I, J, K=$ 0,1 . Local correlation matrices are computed as

$$
C_{i j k, I J K ; e}=\int_{e} \phi_{i} \phi_{j} \phi_{k} \Phi_{I} \Phi_{J} \Phi_{K} d x d y d z .
$$

where the $\Phi$ 's are coarse $1 D$ shape functions. Using fine scale GLL points for integration, we have

$$
C_{I J K, i j k ; e} \simeq \Phi_{I}\left(t_{i}\right) \Phi_{J}\left(t_{j}\right) \Phi_{K}\left(t_{k}\right) m_{i j k ; e} .
$$

Vandermonde matrix

$$
B_{I J K, i j k}=\Phi_{I}\left(t_{i}\right) \Phi_{J}\left(t_{j}\right) \Phi_{K}\left(t_{k}\right)
$$


of size $8 \times n^{3}$ is computed once and stored.

Restriction of the global residual $r_{\mathcal{N}}$ starts by scaling it by the inverted mass matrix to form $y_{\mathcal{N}}=r_{\mathcal{N}} / m_{\mathcal{N}}$ and to use (7) to form the local vector $y_{i j k ; e}$. Then, $y_{i j k ; e}$ is used to form the local coarse residual (see (10):

$$
R_{I J K ; e}=\sum_{i=0}^{n} \sum_{j=0}^{n} \sum_{k=0}^{n} B_{I J K, i j k} y_{i j k ; e} m_{i j k ; e} .
$$

Local coarse residual $R_{I J K ; e}$ is gathered to the coarse grid nodes to form the global coarse residual $R$. Coarse preconditioner $\mathcal{P}_{0}$ is then applied to get the global vector $Z=P_{0} R$. Global vector $Z$ is then scattered to form $Z_{I J K ; e}$. We finally prolongate $Z_{I J K ; e}$ as

$$
z_{i j k ; e}=\sum_{I=0}^{1} \sum_{J=0}^{1} \sum_{K=0}^{1} B_{I J K, i j k} Z_{I J K ; e} m_{i j k ; e} .
$$

and scatter $z_{i j k ; e}$ to form its global version $z_{\mathcal{N}}$. Global vector $z_{\mathcal{N}}$ is finally scaled by $1 / m_{\mathcal{N}}$ to form the coarse scale correction $\left.\mathcal{P}^{c} r\right|_{\mathcal{N}}=z_{\mathcal{N}} / m_{\mathcal{N}}$.

\subsubsection{Algebraic multigrid}

An aggregation based algebraic multigrid method is used to obtain an approximate solution of coarse grid system. At first, the coarse grid matrix is computed using finite elements with polynomial order $n=1$. Then, a hierarchy of matrices are constructed using an unsmooth aggregation method [8]. An approximate solution for the coarse grid problem is obtained by the application of a recursive K-cycle [20] along with damped Jacobi smoothing at each level in the hierarchy as described in [8]. Experimental results show that the algebraic multigrid method provides h-independence convergence.

\subsection{Local preconditioner}

An overlapping additive Schwartz approach is used to build the local preconditioner. A set of $N_{E}$ overlapping subdomains is defined. Each subdomain $s$ is an element of the mesh with one layer of nodes overlapping neighbor elements on all its faces (Figure 1), with a total of $(n+3)^{3}$ GLL nodes per subdomain.

The restriction of $r_{\mathcal{N}}$ on subdomain $s$ is denoted by $r_{i j k ; s},-1 \leq i, j, k \leq$ $n+1$. It is computed using a table $I_{l \rightarrow g}^{p}(i j k ; s)$ that allows to scatter global vectors to the vertices of the subdomains:

$$
r_{i j k ; s}=r_{I_{l \rightarrow g}^{p}(i j k ; s)}
$$




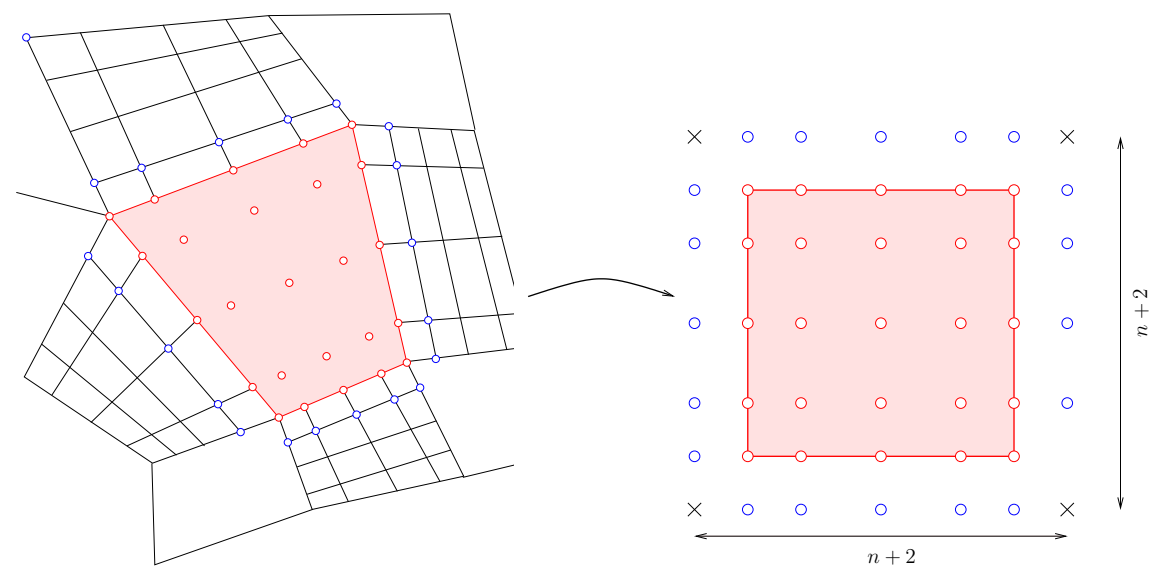

Figure 1: Fine scale stencil for the local preconditioner. Left: GLL nodes shown superimposed on physical elements. Right: topological relationship of GLL nodes on reference element.

The principle is to solve problem (4) on each subdomain i.e. find the fine scale correction $z_{i j k ; b}$ that is the solution of (4) with $r_{i j k ; s}$ as right hand side and with suitable boundary conditions.

We make an assumption that greatly simplifies the computation of fine scale corrections, especially for the case of unstructured meshes. Fine scale corrections are computed without taking into account geometric factors i.e. assuming that hexahedra's faces and edges are aligned with the axis of coordinates $x, y$ and $z$. Each hexahedra is approximated as a parallelogram of dimensions $h_{x}, h_{y}$ and $h_{z}$. This geometric simplification actually allows to compute coarse scale corrections in an efficient way.

Consider a $1 \mathrm{D}$ pencil of size $h_{x}$ with

$$
u(t)=\sum_{i=0}^{n} u_{i} \phi_{i}(t)
$$

with $x=\frac{t+1}{2} h_{x}$ and $u_{i}=u\left(t_{i}\right)$. Assuming $\kappa$ and $c$ constant per element, the $1 \mathrm{D}$ element matrix corresponding to (4) is

$$
K_{i j}=\int_{0}^{h_{x}}\left(\kappa \frac{d \phi_{i}}{d x} \frac{d \phi_{j}}{d x}+c \phi_{i} \phi_{j}\right) d x \simeq \frac{2 \kappa}{h_{x}} \underbrace{\sum_{m=0}^{n}\left(D_{i m} D_{j m} \rho_{m}\right)}_{d_{i j}}+\frac{c h_{x}}{2} \rho_{j} \delta_{i j} .
$$

where $d_{i j}$ is the discrete second derivative. 


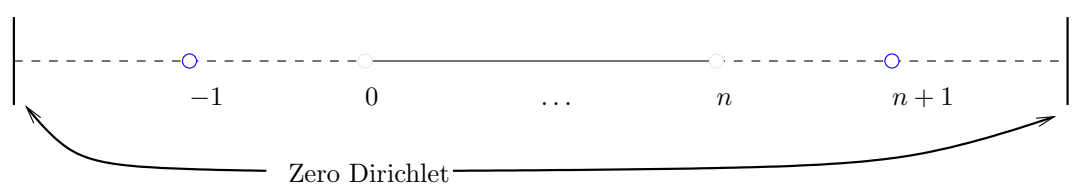

Figure 2: The 1D pencil.

Let's extended the pencil with one GLL vertex on its left (point -1 on Figure 2) and on its right (point $n+1$ on Figure 2). The stiffness operator on the extended domain is computed using standard finite element assembly procedure i.e. adding contribution of vertex -1 and $n+1$ in the finite element matrix. Homogeneous Dirichlet boundary conditions are computed on points -2 and $n+2$ so that the final matrix $l_{i j},-1 \leq i, j \leq n+1$ is invertible. The $1 \mathrm{D}$ finite element problem is finally written as

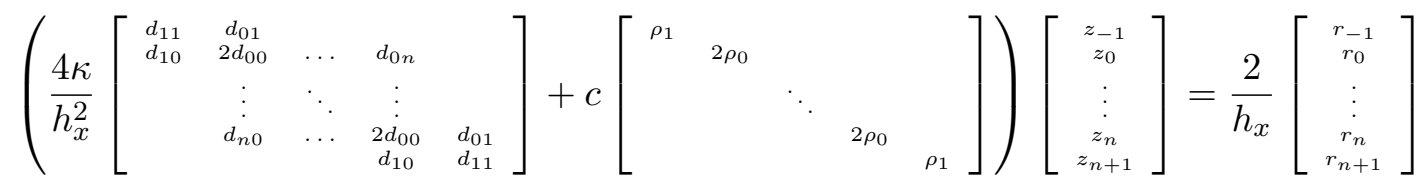

or in matrix form

$$
\left(\frac{4 \kappa}{h_{x}^{2}} K+c M\right) z=\frac{2}{h_{x}} r \quad \rightarrow \quad\left(\frac{4 \kappa}{h_{x}^{2}} M^{-1} K+c I\right) z=\frac{2}{h_{x}} M^{-1} r .
$$

Matrix $L=M^{-1} K$ is diagonalizable

$$
L=V^{-1} \Lambda V
$$

and has real and positive eigenvalues $\lambda_{i}$. The diagonalization of $L$ leads to the explicit solution:

$$
z=\left[V^{-1}\left(\frac{4 \kappa}{h_{x}^{2}} \Lambda+c I\right)^{-1} V\right] \frac{2}{h_{x}} M^{-1} r .
$$

In $3 \mathrm{D}$, the local subproblem consist in finding the fine scale correction $z_{i j k ; b}$ solution of

$$
4 \kappa \sum_{m=-1}^{n+1}\left(\frac{1}{h_{x}^{2}} L_{i m} z_{m j k ; s}+\frac{1}{h_{y}^{2}} L_{j m} z_{i m k ; s}+\frac{1}{h_{z}^{2}} L_{k m} z_{i j m ; s}\right)+c z_{i j k ; s}=r_{i j k ; s}^{\prime}
$$

with

$$
r_{i j k ; s}^{\prime}=\frac{8}{h_{x} h_{y} h_{z}} \frac{1}{M_{i i} M_{j j} M_{k k}} r_{i j k ; s}
$$


It is then possible to compute the solution as we did in 1D:

$$
\begin{array}{r}
z_{i j k ; s}=\sum_{d=-1}^{n+1} \sum_{e=-1}^{n+1} \sum_{f=-1}^{n+1} V_{i d}^{-1} V_{j e}^{-1} V_{f k}^{-1} \frac{1}{4 \kappa\left(\frac{\lambda_{d}}{h_{x}^{2}}+\frac{\lambda_{e}}{h_{y}^{2}}+\frac{\lambda_{f}}{h_{z}^{2}}\right)+c} \\
\sum_{a=-1}^{n+1} \sum_{b=-1}^{n+1} \sum_{c=-1}^{n+1} V_{a d} V_{b e} V_{c f} r_{a b c ; s}^{\prime} .
\end{array}
$$

The fine correction for subdomain $s$ is added to the fine preconditioner

$$
\left.\left.\mathcal{P}^{f}\right|_{I_{l \rightarrow g}^{p}(i j k ; s)} \leftarrow \mathcal{P}^{f}\right|_{I_{l \rightarrow g}^{p}(i j k ; s)}+z_{i j k ; s}, \quad i, j, k \in[-1, n+1]^{3}
$$

\section{Implementation}

Our implementation uses OCCA which is a novel approach that includes a unified kernel language that expands to multiple different threading languages [17] that has recently been demonstrated to be an effective platform for implementing discretizations of hyperbolic problems [9, 18, 19]. The two dominant cost kernels are the computation of residual $r_{i j k ; e}$ and the fine grid preconditioner $z_{i j k ; s}$ (see (12)).

General purpose parallel programming on GPUs allow a two-level parallelism. GPU devices have a large number of multiprocessing units that can run threads. Threads are organized in blocks and whole thread blocks are executed by a multiprocessing unit. The global device memory of the GPU is accessible by all the threads of all blocks. Some memory is only accessible by a given block of threads (shared memory) and has relatively low latency compared to global device memory. In our implementation, each element is assigned to a thread block and residual calculations at each GLL node are assigned to one thread.

\subsection{An OCCA kernel for computing $r_{i j k ; e}$}

Algorithm 2 describes our implementation for computing $r_{i j k ; e}$ : variables with superscript $s$ implies that the data is stored in on-chip shared memory accessible to the threads processing element $e$.

Parameters $\kappa$ and $c$ that are element-based are copied in shared memory, as well as the derivative matrix $D_{i j}$ and the current solution $u$. Then, $(n+1)^{3}$ threads are launched in order to compute (i) derivatives of $u(2 \times 3 \times(n+1)$ 


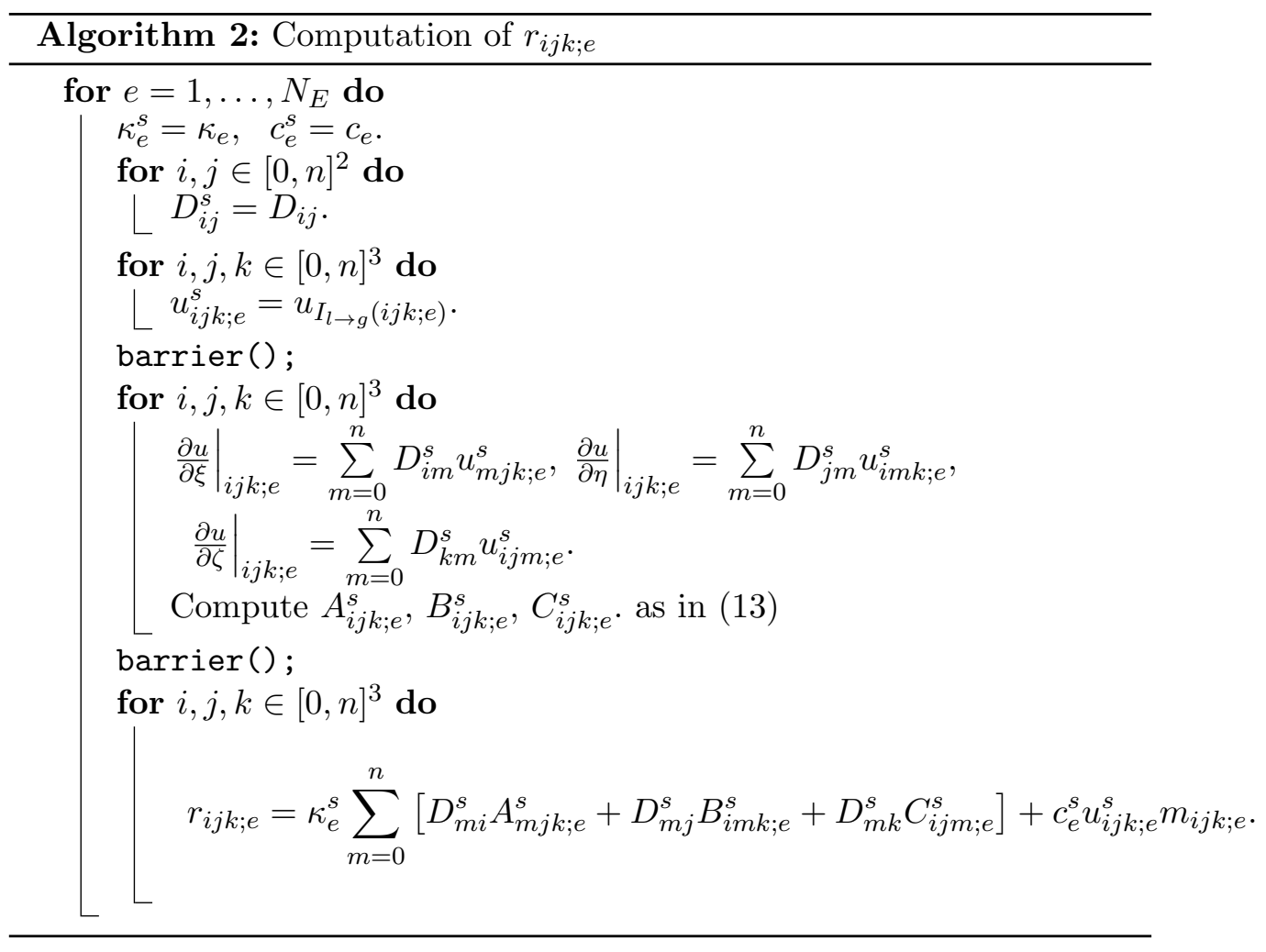


operations per thread), (ii) gradients

$$
\begin{aligned}
& A_{i j k: e}^{s}=\left[\left.G_{i j k ; e}^{1} \frac{\partial u}{\partial \xi}\right|_{i j k ; e}+\left.G_{i j k ; e}^{2} \frac{\partial u}{\partial \eta}\right|_{i j k ; e}+\left.G_{i j k ; e}^{3} \frac{\partial u}{\partial \zeta}\right|_{i j k ; e}\right], \\
& B_{i j k: e}^{s}=\left[\left.G_{i j k ; e}^{2} \frac{\partial u}{\partial \xi}\right|_{i j k ; e}+\left.G_{i j k ; e}^{4} \frac{\partial u}{\partial \eta}\right|_{i j k ; e}+\left.G_{i j k ; e}^{5} \frac{\partial u}{\partial \zeta}\right|_{i j k ; e}\right], \\
& C_{i j k: e}^{s}=\left[\left.G_{i j k ; e}^{3} \frac{\partial u}{\partial \xi}\right|_{i j k ; e}+\left.G_{i j k ; e}^{5} \frac{\partial u}{\partial \eta}\right|_{i j k ; e}+\left.G_{i j k ; e}^{6} \frac{\partial u}{\partial \zeta}\right|_{i j k ; e}\right] .
\end{aligned}
$$

$(3 \times 5$ operations per thread). Finally, the residual is computed at every GLL node $(2 \times 3 \times(n+1)+3$ operations per thread). Two barriers separate the three main parts of the algorithm. The maximum number of threads per thread block in CUDA is currently 1024, our kernel can only be used up to $n=9$ for which $(n+1)^{3}=1000$. In OpenCL the number of workitems per work-group is limited to 256 on AMD GPUs and consequently we are limited to $n=5$ in that case. The total number of operations $O_{R}$ for computing $r_{i j k ; e}$ is

$$
O_{R}=N_{E} \times\left[12 \times(n+1)^{4}+18 \times(n+1)^{3}\right] .
$$

Memory bandwidth quantifies the rate of throughput of data transfer between GPU and the GPU global device memory. In modern GPU architectures, a typical value for the memory bandwidth is $250 \mathrm{~GB} / \mathrm{sec}$ i.e. about 75 billion floats per second. The total number of floating point numbers transferred from the global memory to the shared memory here is

$$
B_{R}=N_{E} \times\left[10 \times(n+1)^{3}+(n+1)^{2}+2\right]
$$

where the dominant term $10 \times N_{E} \times(n+1)^{3}$ correspond (per GLL point) to the 7 geometric factors $G_{i j k ; e}^{m}, 1 \leq m \leq 6$ and the weighted masses $m_{i j k ; e}$, to the solution $u_{i j k ; e}$, to the residual itself $r_{i j k ; e}$ and to the table $I_{l \rightarrow g}(i j k ; e)$ that is used for scattering $u_{\mathcal{N}}$.

At this point, it is interesting to look at the ratio $O_{R} / B_{R}$ Any modern GPU can theoretically deliver over one TeraFlop while its bandwidth is limited to $250 \mathrm{~GB} / \mathrm{sec}$. This means that the value $O_{R} / B_{R}$ should be over 20 to ensure that the computation is not limited by bandwidth limitations. Here, ratio $O_{R} / B_{R}$ is equal to seven for $n=4$ : we expect the performances of our kernel to be strongly limited by the GPU bandwidth, especially at low orders. It is therefore interesting to consider an alternative approach where the seven geometric factors are computed on the fly. In this case, the 
only geometric data that have to be shipped to the GPU are the position of the vertices of the mesh as well as the connectivity table of the hexahedra. More operations are required to compute the geometric factors: more precisely, 242 floating point operations per GLL node are required to compute all seven geometric factors. In what follows, $O_{R}$ will be computed in two different manners, whether the extra computations that have been done for computing geometric factors are or are not not taken into account. In this new scheme, memory bandwidth is indeed reduced to

$$
B_{R}=N_{E} \times\left[3 \times(n+1)^{3}+(n+1)^{2}+2\right]
$$

\subsection{An OCCA kernel for computing $z_{i j k ; s}$}

The local preconditioning step that computes $z_{i j k ; s}$ is the remaining expensive part of the algorithm. Each subdomain $s$ is assigned to a thread block. Then, $(n+3)^{3}$ threads are launched in every block in order to compute $z_{i j k ; s}$, which limits the order to $n=7$ for CUDA and $n=3$ for OpenCL. Equation (12) explicitly provides a formula for $p_{i j k ; s}$. The most significant computations are the six successive products with the left and right eigenvectors $V^{-1}$ and $V$ of the 1D operator. More precisely, the total number of operations $O_{P}$ for computing $p_{i j k ; s}$ is

$$
O_{P}=N_{E} \times\left[6 \times(n+3)^{4}+15 \times(n+3)^{3}\right] .
$$

Geometric factors are not loaded by this kernel, which implies that the total amount of data that is transferred is reduced. Vectors $z_{i j k ; b}, r_{i j k ; b}$ as well as the scattering table $I_{l \rightarrow g}^{p}(i j k ; b)$ are the three large local vectors that have to be transferred from the global memory to the shared memory.

$$
B_{P}=N_{E} \times\left[3 \times(n+3)^{3}+4 \times(n+3)^{2}\right] .
$$

The ratio $O_{P} / B_{P}$ is already large at low orders to expect good performances of the kernel for any $n$.

\subsection{A strategy for computing the preconditioning step}

We have shown that the most intensive part of the computation is the evaluation of the local preconditioner $z_{i j k ; s}$. In an additive Schwartz procedure, coarse and fine preconditioners can be computed independently and summed afterwards. Here, we propose to compute the coarse scale preconditioner on the CPU while computing the fine scale part on the GPU. At low orders, we expect that the computation of the coarse scale preconditioner on the $\mathrm{CPU}$ will hide the computation of the fine scale preconditioner on the 


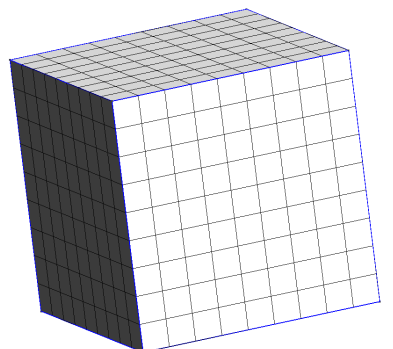

Mesh 1

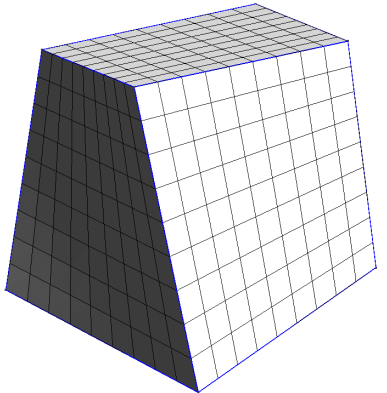

Mesh 2

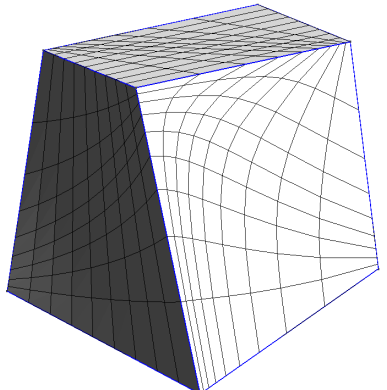

Mesh 3

Figure 3: Three meshes. Left: cube domain meshed with uniform elements. Center: nearly uniform elements on distorted cube domain. Right: strongly distorted elements forming mesh for distorted cube.

GPU. At higher order, the fine scale preconditioner will be more expensive and will hide the computation of the coarse scale part. There may exist a sweet spot where both are taking equivalent time.

\section{Results}

\subsection{Poisson problem}

We choose the parameters in our model problem (3) in order to turn it into a Poisson equation. We choose $\kappa=1, c=0$ and $s=1$.

\subsubsection{Analysis of the two scale strategy}

In this section, we investigate the proposed numerical scheme. Three coarse meshes with $N_{E}=8^{3}$ hexes are considered (see Figure 3 ) and uniformly refined up to $N_{E}=128^{3}$. A polynomial order $n=3$ is used for the computations. The strategy that has been chosen for preconditioning is $h$-optimal: the number of CG iterations is asymptotically stable for all meshes. If only the fine scale preconditioner is used, the number of iterations doubles when the mesh size is divided by two. It must be noted that the computation of $\mathcal{P}^{c}$ (on the CPU) is fully hidden by the computation of $\mathcal{P}^{f}$ (on the GPU) for $n>2$. The coarse scale preconditioner has a zero cost for a large benefit.

Note that the number of CG iterations increases with polynomial order: for mesh 2 at level of refinement $32^{3}$ and for $n=7$, the number of $\mathrm{CG}$ iterations grows to 23. This is due to the fact that the size of the overlap decreases while $n$ grows. A larger overlap would fix that issue [21]. 


\begin{tabular}{|c|c|c|c|c|c|}
\hline $\begin{array}{c}\text { DOF count } \\
N\end{array}$ & $\begin{array}{c}8^{3} \\
1.510^{4}\end{array}$ & $\begin{array}{c}16^{3} \\
1.110^{5}\end{array}$ & $\begin{array}{c}32^{3} \\
9.110^{5}\end{array}$ & $\begin{array}{c}64^{3} \\
7.110^{6}\end{array}$ & $\begin{array}{c}128^{3} \\
5.710^{7}\end{array}$ \\
\hline & \multicolumn{5}{|c|}{ Two scale strategy: $\mathcal{P}=\mathcal{P}^{c}+\mathcal{P}^{f}$} \\
\hline Mesh 1 & 10 & 11 & 13 & 13 & 13 \\
\hline Mesh 2 & 10 & 13 & 15 & 15 & 15 \\
\hline \multirow[t]{2}{*}{ Mesh 3} & 12 & 18 & 21 & 21 & 21 \\
\hline & \multicolumn{5}{|c|}{ Fine scale preconditioner only $\mathcal{P}=\mathcal{P}^{f}$} \\
\hline Mesh 1 & 10 & 16 & 28 & 52 & 103 \\
\hline Mesh 2 & 13 & 20 & 32 & 60 & 118 \\
\hline Mesh 3 & 14 & 23 & 41 & 94 & 185 \\
\hline
\end{tabular}

Table 1: Number of Conjugate Gradient iterations as a function of the preconditioning strategy.

\subsubsection{Performances of the kernels}

We consider here the $32^{3}$ version of Mesh 2 (Figure 3) . Table 2 present flops counts and bandwidth results for different polynomial orders for a uniform mesh of $32^{3}$ elements. Quantities with a prime refer to the kernels for which the geometric factors were computed on the fly. In Table 2, two numbers are given in the row relative to $r_{i j k ; e}^{\prime}$ 's flop count. The first one does not take into account the operations that are required to compute the geometry factors while the second number adds to the flop count $N_{E} \times$ $\left[242 \times(n+1)^{3}\right]$ floating point operations which correspond to the on the fly computation of the 7 geometric factors.

It is interesting to see that the difference in wall clock time between the two approaches is below measurement error. Computing geometric factors in an element is relatively floating point intensive: it consist essentially of loading the 24 coordinates of the eight vertices on registers and performing 242 floating point operations required to compute the geometric factors $G_{i j k ; e^{m}}, 1 \leq m \leq 6$ and $m_{i j k ; e}$ that are themselves stored on registers. The specific GPU that has been used has a very high peak floating point performance (over five teraflops in single precision). Computing geometric factors is done a a rate that is close to the peak performance of the machine while computing the rest of the kernel $r_{i j k ; e}^{\prime}$ requires memory access that are bounded by bandwidth. In the case of kernel $r_{i j k ; e}$, loading geometric factors requires bandwidth: the GPU stalls quickly at about $150 \mathrm{~GB} / \mathrm{sec}$ and so does the flop count. If geometric factors are taken into account in the flop count, the performances of this kernel climb to over one teraflops!

We have done the same computations on a different GPU (see Table 3 ) 


\begin{tabular}{r|rrrrrr}
\hline & $n=2$ & $n=3$ & $n=4$ & $n=5$ & $n=6$ & $n=7$ \\
\hline$N$ & $2.710^{5}$ & $9.110^{5}$ & $2.110^{6}$ & $4.110^{6}$ & $7.110^{6}$ & $1.110^{7}$ \\
\hline$r_{i j k ; e}($ GFLOPs) & 70 & 145 & 227 & 289 & 325 & 362 \\
$r_{i j k ; e}^{\prime}$ (GFLOPs) & $75 / 336$ & $135 / 763$ & $250 / 1050$ & $272 / 1020$ & $286 / 1050$ & $293 / 1050$ \\
$z_{i j k ; s}$ (GFLOPs) & 370 & 371 & 408 & 453 & 421 & 373 \\
\hline$r_{i j k ; e}(\mathrm{~GB} / \mathrm{sec})$ & 59 & 101 & 135 & 150 & 150 & 150 \\
$r_{i j k ; e}^{\prime}(\mathrm{GB} / \mathrm{sec})$ & 32 & 48 & 75 & 71 & 66 & 61 \\
$z_{i j k ; s}(\mathrm{~GB} / \mathrm{sec})$ & 96 & 80 & 76 & 74 & 61 & 49 \\
\hline Wall Time $(\mathrm{sec})$ & 0.10 & 0.12 & 0.23 & 0.34 & 0.53 & 0.83 \\
Wall Time' (sec) & 0.11 & 0.12 & 0.22 & 0.34 & 0.52 & 0.80 \\
\hline
\end{tabular}

Table 2: Performances of the two expensive kernels $r_{i j k ; e}$ and $z_{i j k ; s}$ on a NVIDIA GTX 980 GPU using OpenCL. A uniform mesh of $32^{3}$ hexahedra was used for the test. Apostrophes indicate that geometric factors were computed on the fly. In row relative to $r_{i j k ; e}^{\prime}$, the two numbers correspond whether the cost cof computing geometrical factors was not or was taken into account in $O_{R}$.

with a lower throughput. Kernel $r_{i j k ; e}^{\prime}$ is slightly slower than kernel $r_{i j k ; e}$.

\subsection{Heat equation}

We choose the parameters in out model problem (3) in order to turn it into a heat equation. A backward Euler scheme is used to advance in time. We choose $\kappa=10^{-2}\left[\mathrm{~m}^{2} / \mathrm{sec}\right]$ (aluminium), a time step of $\Delta t=0.04$ seconds and $c=1 / \Delta t$. The source term in (3) is chosen as $r=u_{\mathcal{N}}^{t-\Delta t} / \Delta t+Q /\left(\rho c_{p}\right)$ where $u_{\mathcal{N}}^{t-\Delta t}$ is the solution at previous time step, $Q=1000[W]$ is a volume heat source $\rho=7000\left[\mathrm{~kg} / \mathrm{m}^{3}\right]$ and $c_{P}=0.8$. The volumetric heat source $Q(x, y, z)$ is moved while time is advancing (see Figure 4).

The geometry and the mesh that we consider are presented in Figure 4 We solved 70 time steps of the discretized heat equation on this mesh using different devices (GPU and CPU) and using different threading systems (CUDA, OpenCL, and OpenMP). All computations are performed in single precision arithmetic, either on an NVIDIA (c) GTX 980 GPU or on a 8 core Intel (c) Core $^{\mathrm{TM}}$ i7-5960X CPU @ 3.00GHz that has a theoretical peak performance of about 300 GFLOPS. Results are compiled in Table 4 . Geometrical factors were always computed on the fly and flop count takes into account their computation. Thanks to OCCA, the computations were done both on the GPU and on the CPU using common computational kernels. CPU to GPU speedups of around 10 were observed on all computations: this is about the ratio of memory bandwidths between GPU (200 GB/sec) and the CPU (20 GB/sec). Higher speedups were obtained at higher or- 


\begin{tabular}{c|cccccc}
\hline & $n=2$ & $n=3$ & $n=4$ & $n=5$ & $n=6$ & $n=7$ \\
\hline$N$ & $2.710^{5}$ & $9.110^{5}$ & $2.110^{6}$ & $4.110^{6}$ & $7.110^{6}$ & $1.110^{7}$ \\
\hline$r_{i j k ; e}($ GFLOPs) & 45 & 117 & 194 & 234 & 227 & 287 \\
$r_{i j k ; e}^{\prime}$ (GFLOPs) & 28 & 68 & 91 & 106 & 105 & 112 \\
$z_{i j k ; s}$ (GFLOPs) & 313 & 296 & 285 & 380 & 276 & 371 \\
\hline$r_{i j k ; e}(\mathrm{~GB} / \mathrm{sec})$ & 38 & 82 & 115 & 121 & 104 & 118 \\
$r_{i j k ; e}^{\prime}(\mathrm{GB} / \mathrm{sec})$ & 12 & 24 & 27 & 28 & 24 & 23 \\
$z_{i j k ; s}(\mathrm{~GB} / \mathrm{sec})$ & 81 & 64 & 53 & 62 & 40 & 48 \\
\hline Wall Time $(\mathrm{sec})$ & 0.10 & 0.13 & 0.26 & 0.39 & 0.67 & 0.92 \\
Wall Time' (sec) & 0.11 & 0.14 & 0.29 & 0.46 & 0.79 & 1.13 \\
\hline
\end{tabular}

Table 3: Performances of the two dominant cost kernels $r_{i j k ; e}$ and $z_{i j k ; s}$ on a NVIDIA K40 GPU using CUDA. A uniform mesh of $32^{3}$ hexahedra was used for the test. Apostrophes indicate that geometric factors were computed on the fly.

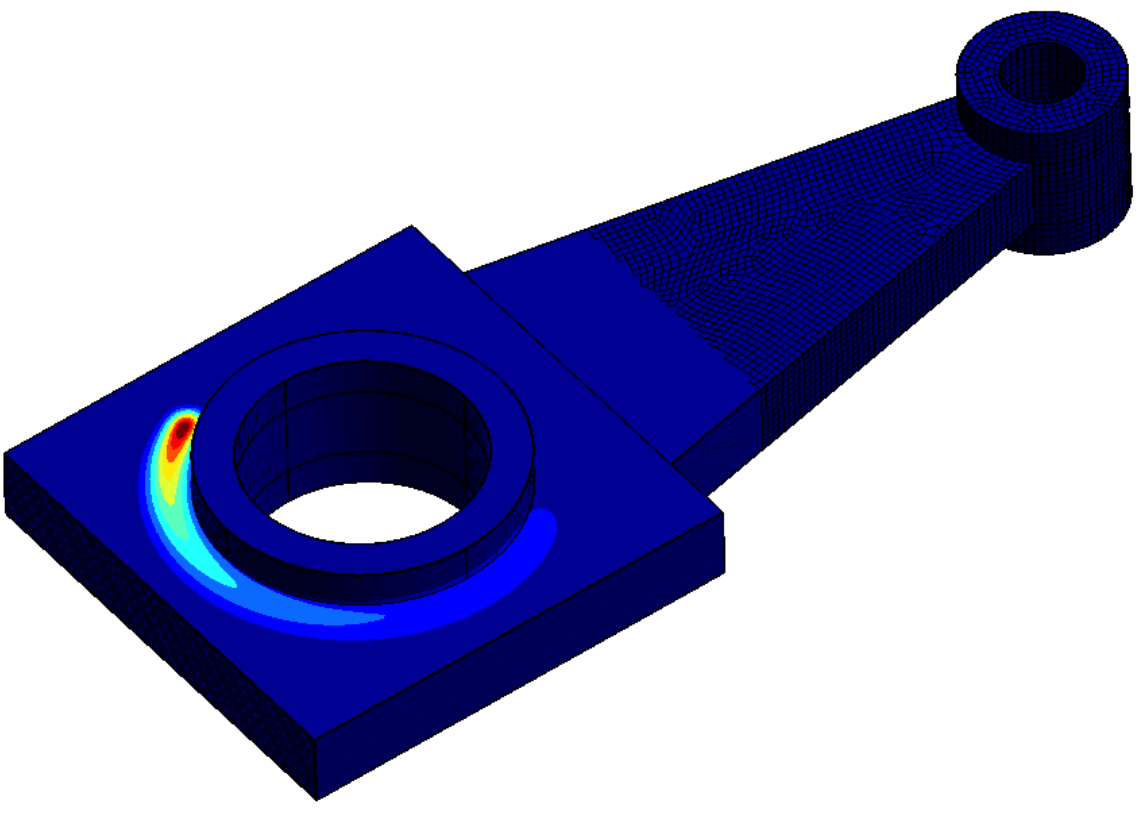

Figure 4: Rod geometry considered for the heat equation with the hex-mesh of 62540 hexahedra.. Temperature field is shown at iteration 70 which correspond to $t=2.8$ seconds. The motion of the heat source is visible on the plot. Mesh is visible on the right part of the plot 


\begin{tabular}{|c|c|c|c|c|c|c|}
\hline & $n=2$ & $n=3$ & $n=4$ & $n=5$ & $n=6$ & $n=7$ \\
\hline device mem.(MB) & 178 & 370 & 587 & 1147 & 1796 & 2660 \\
\hline$N$ & $5.310^{5}$ & $1.710^{6}$ & $4.110^{6}$ & $8.010^{6}$ & $13.810^{6}$ & $21.910^{6}$ \\
\hline \multicolumn{7}{|c|}{ Total wall time (sec) for 70 time steps } \\
\hline CUDA(GPU) & 13.4 & 14.8 & 32.1 & 53.6 & 90.3 & 134.9 \\
\hline OpenCL(GPU) & 12.2 & 13.9 & 31.8 & 49.9 & 83.0 & 126.3 \\
\hline OpenMP (16 threads) & 100.7 & 127.4 & 355.1 & 509.9 & 901.5 & 1393.5 \\
\hline OpenCL(16 threads) & 102.7 & 156.2 & 384.2 & 479.7 & 1052.1 & 1168.5 \\
\hline \multicolumn{7}{|c|}{ Performance in GFLOPs of $r_{i j k ; e}^{\prime}$} \\
\hline CUDA(GPU) & 674.0 & 923.0 & 926.0 & 874.0 & 806.0 & 795.0 \\
\hline OpenCL(GPU) & 1140.0 & 1660.0 & 1690.0 & 1390.0 & 1380.0 & 1310.0 \\
\hline OpenMP (16 threads) & 24.5 & 68.8 & 59.3 & 58.5 & 57.8 & 90.0 \\
\hline OpenCL $(1$ & 30.2 & 39.3 & 42.4 & 42.9 & 43.0 & 206.0 \\
\hline \multicolumn{7}{|c|}{ Performance in GFLOPs of $z_{i j k ; e}$} \\
\hline CUDA & 479.0 & 533.0 & 492.0 & 535.0 & 479.0 & 591.0 \\
\hline OpenCL(GPU) & 422.0 & 385.0 & 457.0 & 470.0 & 445.0 & 478.0 \\
\hline OpenMP (16 threads) & 27.9 & 40.4 & 38.9 & 63.9 & 49.4 & 51.5 \\
\hline OpenCL(16 threads) & 23.7 & 26.6 & 27.4 & 82.6 & 28.8 & 28.5 \\
\hline \multicolumn{7}{|c|}{ Bandwidth in GB/sec for $r_{i j k ; e}^{\prime}$} \\
\hline CUDA(GPU) & 56.9 & 74.0 & 70.9 & 64.2 & $\overline{56.9}$ & 54.1 \\
\hline OpenCL(GPU) & 96.1 & 133.0 & 129.0 & 102.0 & 97.7 & 89.9 \\
\hline OpenMP(16 threads) & 2.1 & 5.5 & 4.5 & 4.3 & 4.1 & 6.1 \\
\hline OpenCL(16 threads) & 2.5 & 3.1 & 3.2 & 3.1 & 3.0 & 14.0 \\
\hline \multicolumn{7}{|c|}{ Bandwidth in GB/sec for $z_{i j k ; e}$} \\
\hline CUDA(GPU) & 125.0 & 116.0 & 91.4 & 87.2 & 69.5 & 77.2 \\
\hline OpenCL(GPU) & 110.0 & 83.4 & 84.9 & 76.6 .0 & 64.6 & 62.5 \\
\hline OpenMP (16 threads) & 7.2 & 8.7 & 7.2 & 10.4 & 7.2 & 6.7 \\
\hline OpenCL(16 threads) & 6.2 & 5.8 & 5.1 & 13.5 & 4.2 & 3.7 \\
\hline
\end{tabular}

Table 4: Overall timings and performances for different devices and threading systems. 
ders thanks to the more computationally intensive nature of higher order computations.

Wall clock times essentially depend on the device: CUDA and OpenCL give similar performances on the GPU. On the CPU, both OpenMP and OpenCL were run on 16 threads using the hyper-threading capacities of our 8-core processor.

Global performance of the code is similar on the GPU, whether OpenCL or CUDA is used as threading system. OpenCL is indeed slightly more efficient, especially for computing the residual.

On the CPU, OpenMP outperforms OpenCL, except for $n=5$ and $n=7$ where either $r_{i j k ; e}^{\prime}(n=7)$ or $z_{i j k ; e}(n=5)$ shows up extraordinary performances with OpenCL. At those respective orders, both the kernels act on array of size $8 \times 8 \times 8$. This array size being an exact fraction of a cache line, memory bandwidth is essentially doubled, reaching about $14 \mathrm{~GB} / \mathrm{sec}$. Note that padding our arrays to a size that is a power of two could certainly improve performances at all orders. OpenCL seems to be better at loop vectorization as well, both on CPU and on GPU.

\section{Conclusions}

In this paper, we have shown that implicit time-steps with over 20 millions of degrees of freedom in a few seconds was possible on an off-the-shelf $\$ 500$ GPU. One of the important features of the method is the two-scale preconditioner with its two parts that can be computed simultaneously on the host and the device.

Another important aspect that we have pointed out, and that significantly differs from the usual way of dealing with finite element codes, is that it is more interesting to re-compute quantities like geometrical factors on the fly than to store them in global memory. This is essentially because GPU computing is more memory bound when compared to CPU computing when considering bandwidth available to each processing element. Therefore, it is more important to reduce the amount of data that is transferred from the global memory on a GPU than on a CPU.

Finally, the use of the common kernel language OCCA allows us to change the device and the thread model in a very simple fashion i.e. using the same code for all platforms. The OCCA kernel language is essentially a "C" language with extra decorations, which makes it very readable to computational scientists.

One order of magnitude speedups is observed between the code running on the CPU and on the GPU. In both cases, we are able to use about $10 \%$ 
of the peak performance of the device, which is to our best knowledge, close to the best one can get.

This paper is essentially about a fast Poisson solver. On the one hand the Poisson equation solver is a rather simple proxy application, but on the other hand a Poisson solver is the building block of more complex models such as incompressible Navier-Stokes equations or acoustics equations. In further work, non-conforming meshes as well as incompressible fluids will be considered.

\section{Acknowledgements}

TW \& RG acknowledge partial support for this research from DOE and ANL (ANL Subcontract No. 1F-32301 on DOE grant No. DE-AC0206CH11357), as well as ONR (grant No. N00014-13-1-0873).

[1] Tristan Carrier Baudouin, Jean-François Remacle, Emilie Marchandise, François Henrotte, and Christophe Geuzaine. A frontal approach to hex-dominant mesh generation. Advanced Modeling and Simulation in Engineering Sciences, 1(1):1-30, 2014.

[2] Steven E Benzley, Ernest Perry, Karl Merkley, Brett Clark, and Greg Sjaardama. A comparison of all hexagonal and all tetrahedral finite element meshes for elastic and elasto-plastic analysis. In Proceedings, 4th International Meshing Roundtable, volume 17, pages 179-191. Sandia National Laboratories Albuquerque, NM, 1995.

[3] Cris Cecka, Adrian J Lew, and Eric Darve. Assembly of finite element methods on graphics processors. International journal for numerical methods in engineering, 85(5):640-669, 2011.

[4] Michel O Deville, Paul F Fischer, and Ernest H Mund. High-order methods for incompressible fluid flow, volume 9. Cambridge University Press, 2002.

[5] Paul F Fischer. An overlapping Schwarz method for spectral element solution of the incompressible Navier-Stokes equations. Journal of Computational Physics, 133(1):84-101, 1997.

[6] Paul F Fischer, HM Tufo, and NI Miller. An overlapping Schwarz method for spectral element simulation of three-dimensional incompressible flows. In Parallel Solution of Partial Differential Equations, pages 159-180. Springer, 2000. 
[7] Zhisong Fu, T James Lewis, Robert M Kirby, and Ross T Whitaker. Architecting the finite element method pipeline for the gpu. Journal of computational and applied mathematics, 257:195-211, 2014.

[8] Rajesh Gandham, Ken Esler, and Yongpeng Zhang. A GPU accelerated aggregation algebraic multigrid method. Computers and Mathematics with Applications, 68(10):1151-1160, 2014.

[9] Rajesh Gandham, DS Medina, and Timothy Warburton. GPU Accelerated discontinuous Galerkin methods for shallow water equations. Communications in Computational Physics, 2015.

[10] Christophe Geuzaine and Jean-François Remacle. Gmsh: A 3-D finite element mesh generator with built-in pre-and post-processing facilities. International Journal for Numerical Methods in Engineering, 79(11):1309-1331, 2009.

[11] Markus Geveler, D Ribbrock, D Göddeke, P Zajac, and S Turek. Towards a complete fem-based simulation toolkit on gpus: Unstructured grid finite element geometric multigrid solvers with strong smoothers based on sparse approximate inverses. Computers \& Fluids, 80:327-332, 2013.

[12] Dominik Göddeke. Fast and accurate finite-element multigrid solvers for PDE simulations on GPU clusters. Logos Verlag Berlin $\mathrm{GmbH}$, 2011.

[13] Dominik Göddeke, Robert Strzodka, Jamaludin Mohd-Yusof, Patrick McCormick, Sven HM Buijssen, Matthias Grajewski, and Stefan Turek. Exploring weak scalability for fem calculations on a gpu-enhanced cluster. Parallel Computing, 33(10):685-699, 2007.

[14] Koen Hillewaert, Nicolas Chevaugeon, Philippe Geuzaine, and JeanFrançois Remacle. Hierarchic multigrid iteration strategy for the discontinuous Galerkin solution of the steady Euler equations. International journal for numerical methods in fluids, 51(9-10):1157-1176, 2006.

[15] Matthew G Knepley and Andy R Terrel. Finite element integration on gpus. ACM Transactions on Mathematical Software (TOMS), 39(2):10, 2013.

[16] GR Markall, A Slemmer, DA Ham, PHJ Kelly, CD Cantwell, and SJ Sherwin. Finite element assembly strategies on multi-core and many- 
core architectures. International Journal for Numerical Methods in Fluids, 71(1):80-97, 2013.

[17] David S Medina, Amik St-Cyr, and T Warburton. OCCA: A unified approach to multi-threading languages. arXiv preprint arXiv:1403.0968, 2014.

[18] David S Medina, Amik St-Cyr, and Timothy Warburton. High-Order Finite-differences on multi-threaded architectures using OCCA. arXiv preprint arXiv:1410.1387, 2014.

[19] A Modave, A St-Cyr, T Warburton, and WA Mulder. Accelerated discontinuous Galerkin time-domain simulations for seismic wave propagation. In 77th EAGE Conference and Exhibition 2015, 2015.

[20] Yvan Notay. An aggregation-based algebraic multigrid method. Electronic Transactions on Numerical Analysis, 37(6):123-146, 2010.

[21] Luca F Pavarino. Additive Schwarz methods for thep-version finite element method. Numerische Mathematik, 66(1):493-515, 1993.

[22] MA Puso and J Solberg. A stabilized nodally integrated tetrahedral. International Journal for Numerical Methods in Engineering, 67(6):841$867,2006$.

[23] Soji Yamakawa and Kenji Shimada. Fully-automated hex-dominant mesh generation with directionality control via packing rectangular solid cells. International journal for numerical methods in engineering, 57(15):2099-2129, 2003. 